\title{
RELACIÓN ENTRE LAS CARACTERÍSTICAS DE LA ADOLESCENTE EMBARAZADA Y LA RESISTENCIA AL CONSUMO DE DROGA ${ }^{1}$
}

Martinez LC, Ferriani MGC. Relación entre las características de la adolescente embarazada y la resistencia al consumo de droga. Rev Latino-am Enfermagem 2004 março-abril; 12(número especial):333-9.

Es un estudio cualitativo, se realizo entrevistas en profundidad a 20 adolescentes embarazadas, que aceptaron participar de la investigación, sobre la relación entre las características de la adolescente embarazada y la resistencia al consumo de droga. Se logro conocer que ellas tuvieron contacto con drogas licitas e ilícitas, así como la decisión para rechazar, dejar o disminuir el consumo; también se identifico el escaso conocimiento que las jovencitas tienen del salud reproductiva y las modificaciones gravídicas en su cuerpo. Para esta población es importante contar con una pareja y su hijo, como sostén para el no consumo de sustancias toxicas que causan adicción.

DESCRIPTORES: adolescencia; adolescente embarazada; resiliencia; adicción; drogadicción en adolescentes; consumo del cigarrillo; enfermería

\section{RELATION BETWEEN CHARACTERISTICS OF PREGNANT ADOLESCENTS AN RESISTANCE TO DRUGS USE}

This is a qualitative study. We interviewed 20 pregnant adolescents, who agreed to participate in this research about the relation between the characteristics of pregnant adolescents and resistance to drugs use. We observed that they had contact will legal and illegal drugs, as well as their decision to reject them, give up or decrease substance use; we also identified a lack of knowledge about reproductive health and body changes. It is important for this population to be able to count on their partner and child, as a support not using addictive toxic substances.

DESCRIPTORS: adolescence; pregnant adolescent; resistance; addiction; substance-related disorders; tobacco use; nursing

\section{RELAÇÃO ENTRE AS CARACTERÍSTICAS DA ADOLESCENTE GRÁVIDA E A RESISTEN CIA CONTRA O CONSUMO DE DROGAS}

Este é um estudo qualitativo. Foram realizadas entrevistas com 20 adolescentes grávidas, que aceitaram participar da pesquisa sobre a relação entre as características da adolescente grávida e a resistência contra o consumo de drogas. Foi observado que tiveram contato com drogas legais e ilegais, e a sua decisão para rejeitá-las, desistir ou diminuir o consumo; também identificamos a falta de conhecimento das jovens sobre saúde reprodutiva e as modificações corporais. Para essa população, é importante poder contar com o parceiro e o filho, como apoio para não consumir substâncias tóxicas aditivas.

DESCRITORES: adolescência; adolescente grávida; resistência; dependência; transtornos relacionados ao uso de substâncias entre adolescentes; consumo de tabaco; enfermagem

\footnotetext{
${ }^{1}$ Las opiniones expresadas en este articulo son de responsabilidad exclusiva de los autores y no representan la posición de la organización donde trabajan o de su administración; ${ }^{2}$ Licenciado en Enfermería, Docente de la Escuela de Enfermería de la Universidad Nacional de Córdoba, Enfermera del Servicio de Neonatologia del Hospital Universitario de Maternidad y Neonatologia, Universidade Nacional de Córdoba; ${ }^{3}$ Docente de la Escuela de Enfermería de Ribeirão Preto, de la Universidad de São Paulo, Centro Colaborador de la OMS para el desarrollo de la investigación en enfermería, correo electrónico: caroline@eerp.usp.br
} 


\section{INTRODUCCIÓN}

La Argentina y la ciudad de Córdoba, inmersas en un clima de conflictos sociales y económicos, donde los problemas de Salud, están afectando a la población, sin límite de frontera; surge como emergente preocupante entre los adolescentes el consumo de Drogas lícitas e ilícitas. La fármaco-dependencia se presenta como un fenómeno que causan adicción. Existe una relación particular con las adolescentes embarazadas; porque están los factores intervinientes que propician el consumo, produciendo daño en el organismo y en la estructura familiar.

Según La Secretaría de Programación para la Prevención de Drogadicción y Lucha Contra el Narcotráfico de la Presidencia de la Nación, de $1999^{(1)}$, informo que 3 de cada 10 personas mayores de 16 años consumen sustancias ilegales.

En un estudio realizado entre 1993 y 1998 por la Fundación CLACYD (Córdoba, Lactancia, Alimentación, Crecimiento y Desarrollo) se estableció que al momento del parto un 13,5\% corresponde a adolescentes menores de 19 años. Esta población necesita de una enfermería capacitada para brindar una atención adecuado; ante un embarazo no programado y/o deseado así como para detectar a tiempo adicciones propias de esta edad ${ }^{(1)}$.

Las adolescentes al conocer su estado de gravidez deben enfrentar problemas entre los cuales, está informarle a su pareja, amigos, familia; además, debe tomar una decisión personal de continuar con su embarazo, más aun cuando ha estado consumiendo drogas, pero ¿existen factores protectores ante esta adversidad?

Por esto se tomo como objeto de estudio: La Relación entre las características de las adolescentes embarazadas, y la resistencia al consumo de drogas de las que concurren al Hospital Universitario de Maternidad y Neonatología, Centro Salud № 28 "Las Violetas" de la Dirección de Atención Primaria de Salud de la Municipalidad. Córdoba. Marzo 2003.

Con los siguientes objetivos:

- Conocer la relación entre las características de las adolescentes embarazadas y la resistencia al consumo de drogas.

- Identificar sus emociones ante los cambios anátomo funcionales y su resistencia al consumo de drogas lícitas e ilícitas.

El propósito es preparar un espacio para que enfermería realice la valoración y el asesoramiento anticipado, a la adolescente embarazada en el auto cuidado, pudiendo abordar de ésta manera la problemáticas de salud desde una concepción de integralidad, a los efectos de reforzar y ayudar a mantener aquellos factores protectores de la madre y el niño en una situación de tantos conflictos.

\section{METODOLOGÍA}

Se trata de un estudio cualitativo realizado en base a entrevistas en profundidad orientadas por una guía de entrevista y realizadas a 20 adolescentes embarazadas que se atendían en dos instituciones de salud de la ciudad de Córdoba, Argentina, quienes aceptaron formar parte de esta investigación.

En este estudio cualitativo, la riqueza esta en las entrevistas cara a cara, la observación y un cuaderno de campo, permitieron llegar a la saturación, análisis e interpretación de los datos ${ }^{(2-3)}$.

Según datos oficiales en la capital de Córdoba, en el año 2000, 534 defunciones entre las edades de 5 a 24 años, de los cuales 177 corresponden a mujeres, Si bien solo se informa 2 muertes por causa del embarazo, parto y puerperio en toda la provincia lo que si es preocupante que para el mismo estudio se han producido en la etapa postnatal 104 muertes. Los datos oficiales no permitió establecer cuántas de estas muertes son prevenibles y corresponden especialmente a las adolescentes entre 14 y 20 años $^{(4)}$.

Para tener una mirada holística en profundidad de la situación planteada se eligieron como escenarios dos instituciones de Salud con perfiles de adolescentes en diferentes situaciones económicas y de educación.

Uno de ellos fue el Hospital Universitario de Maternidad y Neonatología (HUMN) dependiente de la Facultad de Ciencias Médicas de la Universidad Nacional de Córdoba. En un 70\% recibe pacientes con Obra Social o con derivaciones de la provincia para los servicios de obstetricia y neonatología.

La segunda institución fue el Centro Salud № 28 "Las Violetas", dependiente de la Dirección de Atención Primaria de la Salud. Secretaria de Salud. Municipalidad de la Ciudad de Córdoba, que pertenece a la red de $1^{\mathrm{er}}$ nivel de atención, estos son referentes y contrarreferencia de los hospitales en la Capital de la Provincia. La 
prestación es gratuita; pero son insuficientes los recursos materiales y la cobertura.

Cada entrevista fue realizada en total privacidad con las veinte jovencitas que desearon participar en la investigación luego de aceptar la divulgación de los informaciones de manera anónima, lo que le permitió contestar sin temor ni inhibiciones para poder encontrar elementos de resistencia a las drogas.

Se utilizo un cuestionario con preguntas abiertas que permitió abordar los siguientes aspectos: datos demográficos, familiares, de amistad/recreación, obstétricos y experiencia con el consumo de sustancias ilícitas y licitas.

El material obtenido fue clasificado en categorías homogéneas que contenían las siguientes unidades de estudio: Características y conocimiento del embarazo, Conocimiento de las drogas. Identificación del auto cuidado por el abandono del consumo de sustancias ilícitas y licitas (cigarrillo). Relación entre el estado de Gravidez y la resistencia al consumo de drogas.

\section{HALLAZGOS Y PRESENTACIÓN DE LOS CASOS}

Contexto, escenarios y sujetos

En Córdoba la tasa bruta de natalidad es del 17,5 por mil habitantes; el 16,3\% corresponden a madres menores de 20 años $^{(4)}$.

El nivel de instrucción de las jovencitas fueron: 2 analfabetas, 8 de ellas no ha terminado la primaria, 5 completaron la primaria 4 alcanzaron hasta 3 año del ciclo básico común y solo una esta cursando la universidad.

Ninguna de ellas cuenta con una Obra Social; hasta el momento estas jovencitas no han logrado ubicarse en el rol de organizadoras de su hogar; "solo, estoy en casa".

Para cuatro de ellas, este es el $2^{\circ}$ embarazo y para dos el $3^{\circ}$, con un intervalo intergenésico de dos años, 14 fueron primigestas.

Pero ser mamá es un accidente del desconocimiento de su ciclo menstrual o un pedido de la pareja de tener un hijo "para él".

Según la O.M.S. estas adolescentes menores de 18 años, son vulnerables y el riesgo aumenta en la medida que sumas factores; además ser primigesta o tercigesta en plena inmadurez del cuerpo es causa de morbimortalidad en la mujer ${ }^{(5)}$.

Las adolescentes embarazadas y sus vínculos

"Las definiciones tradicionales de la salud de la mujer deben ser ampliadas más allá de los límites de la reproducción y la maternidad, incorporando en ellas los aspectos de salud física y mental del ciclo vital”(6).

En la pubertad se busca; la independencia, este grupo de adolescentes embarazadas manifiestan estar conviviendo con su pareja. Yo me fui a vivir con él..., Estamos viviendo en unas piecitas al fondo de mi casa con mi marido.

La búsqueda de un espacio propio y su ubicación en una familia, motivan a la adolescente a aceptar compartir, con su pareja una habitación o casa como algo suyo. Se siente independiente cuando ha salido de su hogar no así en lo afectivo.

Ellas siempre se refieren a la pareja como su "marido", aunque aun no están casados, pero están viviendo con él desde hace seis meses o más de un año.

En este nuevo hogar las jovencitas aun no asumen su rol; solo manifiestan estar "en casa" con mucho tiempo libre: ahora; en realidad me pidieron que regrese, cuide a mi abuela.

La comunicación a los padres sobre el embarazo despierta en ellos dos reacciones: de aceptación y resignación; estas conductas en los padres depende principalmente de los proyectos y expectativas que ellos tienen para su hija: mi papá se puso loco... yo soy la mas chica.

Otra situación se da en la familia del novio, no aceptan que él hijo asuma su paternidad; también tiene que ver con la construcción del futuro de su hijo o no ella decide no comunicarle:

Aun no le he dicho nada a él..

Las amigas de la infancia pueden continuar o se modifican de acuerdo a las experiencias vivenciadas; cada una hizo su vida, las amigas se alejan por: cambio de domicilio/ocupación y solo están las "conocidas" con las que comparte momentos de recreación. Esto tendría relación con el contexto actual; el individuo está cada vez más solitario, en una sociedad donde la confianza y la honestidad hacia el otro se encuentran en crisis y lleva a desconfiar de todo solo tengo conocidas.

¿Qué esta pasando con mi cuerpo de niña/mamá?

Es importante observar los riesgos socioeconómicos de estas jovencitas por que en su mayoría 
pertenecen a familias donde la información es insuficiente para acceder precozmente a los controles ginecológicos una vez iniciada la pubertad.

Ellas perciben la pérdida de su propia infancia, deben asumir la responsabilidad de su sexualidad para programar los embarazos y evitar el contagio de enfermedades transmisibles; dicen tener conocimiento de los métodos anticonceptivos mencionando como los más conocidos las pastillas, los preservativos... pero no lo utilizan; no los utilice nunca.

Estas jovencitas han iniciado su actividad sexual alrededor de los 14 años, su menarca fue a los 11 años, en mas de una ocasión quedan embarazadas antes de conocer su ciclo menstrual ...me viene todos los meses.

Estas jovencitas manifiestan que estuvieron buscando este embarazo; y ante una amenorrea, realizan un test de gravidez o van a un control obstétrico para realizarse una ecografía, porque: era saberlo por sentir cosas distintas.

El colectivo de las mujeres en edad fértil, conocen el momento para embarazarse, pero estas jovencitas desconocen las posibles complicaciones para la madre y el recién nacido, quien puede necesitar de un equipo de alta complejidad para ser atendido.

Ante la confirmación del embarazo, surgen sus emociones: Me quería morir, lo buscábamos... pero no ahora, Llore llore de alegría.

La adolescente esta pasando por cambios fisiológicos importantes, está modificando su imagen corporal, impacta en su identidad y debe reubicarse en un rol; por lo general está dependiendo económicamente de sus padres o su pareja, asistiendo al colegio, trabajando y participando de los momentos de recreación propia de su edad $^{(7)}$.

Es importante saber, con quienes se cuenta para hacer frente esta situación; por supuesto esta mi marido y mi familia, por que el embarazo trae modificaciones psícofisiológicas; esto tiene que ver con cambios en el aparato circulatorio, digestivo, hormonal, esquelético y de conducta como temores, molestias, ansiedades y miedos.

¡Estar embarazada es algo más ...!!

El embarazo trae sus propias modificaciones psíco-fisiológicas; esto tiene que ver con cambios importantes en el aparato circulatorio, digestivo, hormonal, esquelético. También a medida que el embarazo avanza su cuerpo va cambiando y su piel presenta zonas de mayor pigmentación, en ocasiones presenta en el rostro manchas denominadas cloasma, además tiene cambios de conducta como temores, molestias, ansiedades y los miedos propios del embarazo ${ }^{(5)}$.

Al indagar sobre conocimientos previos sobre el embarazo, por lo general responde no saber nada o contesta: uno va engordando y aumenta el pecho.

Sin diferenciar el nivel de instrucción y edad, todas ellas presentan una desinformación en cuanto a las modificaciones gravídicas. Esto lo relacionan con la vivencia de estar embarazada Te sentís gorda, distinta todo cambia, Sentimental en todo momento.

La mujer en esta etapa necesita cuidados para la recuperación y adaptación; en cuanto a nutrientes, horas de sueño, higiene y vestimenta. Estás adolescentes duermen entre 6 a 13 horas por día. Se observa en ellas un cuidado en su aspecto personal, sus cabellos están limpios al igual que su vestimenta; es sencilla, pero en lo posible buscan combinar los colores, ellas prefieren la ropa suelta o deportiva pero utilizan ropa ajustada y están las que dicen me gusta mostrar la pancita. En cuanto a los alimentos manifiestan comer de todo.

Se puede decir que está el grupo de futura mamá que comen de todo; pero en oposición están las que si les preocupa la ganancias de peso o no cuentan con el recurso suficiente para mantener una dieta equilibrada.

Las jovencitas desconocen la importancia del control prenatal y de los cuidados de salud durante el embarazo; ellas tienen más riesgo de que su embarazo finalice inadecuadamente a diferencia de aquella mujer que empieza el embarazo con un riesgo más bajo, las probabilidades de hipertensión inducida por el embarazo, las anomalías fetales, la amenaza de partos prematuros entre otros, se observa más a menudo en las mujeres menores de 21 años. El uso de teratógenos que pueden causar complicaciones evitables, en esta etapa del desarrollo y crecimiento fetal determinará el futuro del recién nacido, la madre adolescente y su entorno familiar ${ }^{(6-}$ 7).

¡"YO no se nada sobre las drogas"!!

El uso y abuso de sustancias denominadas drogas responde a un complejo fenómeno que involucra las conductas humanas y su aprendizaje, así como la respuesta de la sociedad hacia quien los utiliza. 
En primera instancia se necesitaba conocer que

significado tenia la droga: drogarse es algo malo para la salud, no le doy importancia no significa nada.

Al abordar esta temática con las jovencitas despertaba ciertas reacciones de alerta; en general todas conocían los nombres de las drogas, los diferentes lugares y momentos de consumo en: plazas, bailes, boliches y reuniones de amigos.

El uso de sustancias denominadas drogas, responde a un fenómeno complejo que involucra las conductas humanas y la respuesta de la sociedad hacia quien los utiliza.

Ellas saben lo que más se consume: la fana (pegamento) porro/chala (marihuana), papelito, polvo/ravioles (cocaína) y está el éxtasis al que solo han oído nombrar y las anfetaminas; solo una de ella menciono al cigarrillo (tabaco).

Desde el modelo ético-jurídico, se las clasifica en sustancias legales e ilegales. Así tenemos como legales o licitas: el cigarrillo e ilegales o ilícitas: pegamento, marihuana, cocaína, éxtasis y el uso de fármacos con propósitos no medicamentosos.

Hacen referencia, que el consumo de drogas ilícitas es porque: ellos tienen problemas familiares o se da en boliches y bailes.

Se nota que el consumo se da por conflictos familiares, para divertirse o delinquir y lo realizan; siempre porque les gusta o las veces que tienen ganas...

Las que asisten al Centro Salud № 28 del barrio "Las Violetas" donde el consumo es en la vía pública; esto no es molesto bien... no tiene nada de malo.

Las entrevistadas en la Maternidad Nacional afirmaron: no sabría que hacer, Incomoda, Además ellos están tan drogados que no saben lo que hacen.

A pesar de esta diferencia; ambos grupos han tenido experiencia con amigos/conocidos y compañeros de estudio que eran adictos a estas sustancias.

Al hacer la relación entre las drogas y el posible daño que la misma puede ocasionar al bebe durante el embarazo, ellas dicen que saben muy poco: Afecta en todo; el cerebro, puede salir enfermo, con problemas a los pulmones.

Lo estudiado nos presenta que la cocaína y sus derivados, los opiáceos, fármacos sintéticos, morfina y heroína producen en el recién nacido: síndrome de abstinencia dentro de las primeras horas, con languidez y signos de hiporreflexia muscular, escasa capacidad para la succión y un llanto agudo de irritabilidad.
Por supuesto que he...!!!

Las conductas negativas como el consumo de sustancias nocivas es una invitación para esta comunidad, pero son los mecanismos de auto-cuidado los que resguardan la salud de ellas.

Para estas adolescentes el consumo de tabaco y alcohol no esta incorporada como una droga aunque si puede causar adicción.

Estas jovencitas en algún momento han fumado: hace mucho tiempo, hace mas de dos años, fumaba uno ocasionalmente o lo probé a los 12 años...

Al ir indagando sobre el consumo de cigarrillo el grupo se subdivide entre aquella que mantienen el consumo de cigarrillos pero que han disminuido la cantidad; fumo uno a la mañana y otro a la tarde; las que lo dejaron por el embarazo; por mi bebe mi esposo no quiere que fume y las que solo probaron en alguna ocasión.

¿Qué situaciones te llevo a dejar de fumar?

La resiliencia habla de una combinación de factores que permiten a un niño, a un ser humano, afrontar y superar los problemas y adversidades de la vida ${ }^{(8)}$.

Todos me decían que era malo para mi bebe, un día decidí dejarlo y lo deje.

Ante esta decisión es importante que se tenga alternativas de solución en los momentos de crisis de abstinencia; por momentos me desespero y tengo muchísimas ganas de fumar aunque tengo dinero para comprar la etiqueta, me compro algo dulce.

Además de estos aspectos existen otros motivos que refuerzan el hecho resistir al consumo del cigarrillo; ahora el olor me causa rechazo o nadie fuma a mi alrededor así que ya no fumo.

Este grupo que dejo de fumar, una vez que le confirmaron el embarazo necesitaron de un apoyo emocional, como la madre o el marido para resistirse al gran deseo de fumar.

Algunas de ellas al inicio del embarazo comienzan con náuseas y vómitos, situación que las lleva a no soportar el olor y el sabor del tabaco, a partir de ese momento dejan de fumar y por el apego a su niño toman la decisión abandonar este consumo.

Ellas saben de los riesgos que representa el consumo de cigarrillo durante el embarazo y la lactancia: mi abuelo murió de cáncer a los pulmones o en todo lados te dicen que es malo durante el embarazo. 
Las adolescentes que han disminuido el consumo de cigarrillos conocen de los daños de esta adicción para ellas y su bebe pero el ambiente en el que están conviviendo y el medio resulta una tentación constante ante el aroma del tabaco que invita a fumar; para lograr superar esta etapa consumen caramelos o buscan distraerse realizando diversas tareas.

\section{CONCLUSIÓN}

El análisis de la información recogida en las entrevistas permitió realizar las inferencias que se exponen seguidamente.

Una tendencia en esta generación joven, es iniciar tempranamente con las relaciones sexuales; son madres a los 14 años, interrumpen su educación y no cuentan con una obra social.

En la búsqueda de afecto e independencia aceptan convivir con su pareja; en esta primera etapa ellas son sostenidas económicamente por el "marido".

El individualismo y la desconfianza, de estos momentos de constante violación a los principios de solidaridad y respeto por el otro están modificando estas conducta donde se colocan a las amigas en el plano de "conocidas".

El desarrollo anátomo fisiológico de las adolescentes, y la presencia de la menarca a una edad cada vez más temprana; estaría influyendo en embarazos a una edad tempana. Esta jovencitas desconocen los riesgos y la manera de prevenirlos a través del uso de métodos anticonceptivos y el control prenatal precoz, continuo, completo y de amplia covertura.

Hacer referencia a la droga es hablar de las sustancias ilícitas, además de toda una connotación particular que ocasiona temor a ser identificada como consumidoras, especialmente entre las asistentes al Centro Salud "Las Violetas". En principio se niegan a conceptualizar ¿qué es la droga? pero en el dialogo ellas muestran conocer sobre las características de las sustancias como la marihuana, cocaína; el lugar y frecuencia de su consumo; dos de ellas confirmaron haber fumado marihuana y otra que uso pegamento en una de las esquina del barrio; sus motivos para consumir fueron sus problemas familiares y su soledad. Pero esta nueva experiencia y etapa de su vida les permite mantenerse alejadas de todo consumo.
El grupo sí experimento el consumo del tabaco, en alguna ocasión; un grupo mantiene el consumo en menor cantidad, su resistencia está en poder controlar la disminución del número de cigarrillos que fuman por día.

El grupo que dejo de fumar al momento de embarazarse cuenta con el apoyo de su pareja y familiares cercanos para mantenerse en abstinencia, además de estar constantemente restableciendo el vínculo con su hijo, pero debe tener un soporte externo reforzando su propia fuerza de voluntad para superar los periodos de deseo intenso

Una vez que ellas han confirmado su embarazo, buscado pero no deseado logran establecer un vínculo a través de la aceptación y apoyo de su pareja, familiares y de los amigos.

Esta la capacidad de superar la adversidad del consumo de cigarrillo, pegamento y/o marihuana donde ellas sostienen que un día decidí dejarlo y lo deje, reafirmar que tienen un motivo para mantenerse en esta posición, yo me sentía muy sola pero ahora mi bebé va ser mío.

También están las que tienen características personales para poder superar las presiones y así lo demuestran cuando afirmaron yo dejo de fumar cada vez que me embarazo pero que aun no conocen este potencial para mantenerlo aunque necesitan de apoyo para superar los momentos.

Por el contrario están las que disminuyen el consumo debido a que reconocen los daños que produce en el feto pero que aun no han podido ejercitar esta resistencia; ellas son quizás las que van a necesitar de consejería y apoyo terapéutico externo.

Pero es de destacar en ellas su esfuerzo para desarrollar capacidades para superar esta adversidad por momentos me desespero y tengo muchísimas ganas de fumar aunque tengo dinero para comprar la etiqueta me compro algo dulce o cada vez que tengo un gran deseo de fumar me pongo a hacer cosas y me olvido, verbalizar ayuda a generar una posición de resiliencia.

\section{RECOMENDACIÓN}

El 97\% de los partos en la provincia de Córdoba son institucionales este dato nos hace reflexionar de las oportunidades perdidas que tiene los equipos de Salud para realizar promoción y prevención en la mujer y su familia. 
De acuerdo a los hallazgos y a la conclusión; estas adolescentes embarazadas a tan temprana edad necesitan de una Enfermería formada para la identificación temprana de estos nuevos problemas de adicción y en ese sentido se recomienda:

- Propiciar los estudios de investigación para identificar los factores protectores de las adolescentes embarazadas en estas situaciones de crisis social, económica y de valores.

- Reveer los factores que están determinando que la población femenina embarazada es captada recién en el tercer trimestre.

- Profundizar y ampliar el estudio sobre los conceptos de Salud, Educación, Valores y Proyectos de Vida de los adolescentes en una sociedad Globalizada.

- Analizar los programas de Salud Reproductiva en cuanto a responsabilidad del estado, institución y familia.

- Analizar la cobertura de los recursos materiales y las capacidades personales para la atención de una población informada.

- Identificar los puntos críticos a los efectos de disminuir las oportunidades perdidas de captación de la embarazada adolescente de bajo y alto riesgo.

- Establecer en el control prenatal un Ítems especial para la adolescente embarazada donde este especificado los riesgos de adicción y otras patologías propias de esta etapa y situación.

\section{REFERENCIAS BIBLIOGRÁFICAS}

1. Secretaría de Programación para la Prevención de Drogadicción y Lucha Contra el Narcotráfico (AR). Proyecto de Enseñanza de la Prevención y Atención de Enfermería en la Relación al Uso Indebido de Drogas. Marco referencial y conceptual. Aspectos Globales de la República Argentina. UNC-FCM-EF-CICAD/OEA. Córdoba; 1999. Serie Milenio Sin Droga, $\mathrm{n}^{\circ} 2$.

2. Minayo MCS. Pesquisa Social. Teoría método y creatividad. 20 ed. Petropolis (RJ): Vozes; 2002.

3. Triviños A. Introducción a la investigación en ciencias sociales: la investigación cualitativa en educación. São Paulo (SP): Atlas; 1997.

4. Silvina M. Perfil epidemiológico de las adolescentes embarazadas en Córdoba. [Tesis Maéstria]. Córdoba (C): Escuela de Enfermería/EGCS/FCM/UNC; 1996.

5. Sllber T. Munist Mabel M. Manual de medicina en la adolescencia. Ginebra (SWZ): OPS/OMS; 1992. Serie Paltex para ejecutores de programas de salud, no 20. 1-31 y 250268.
- Fortalecer y promover el asesoramiento de enfermería en los consultorios de control prenatal para planificar cuidados en la pre y post-consulta de la adolescente embarazada.

- Generar programas de capacitación del personal de enfermería en las instituciones de 1ํㅜ $2^{\circ}$ y $3^{\circ}$ nivel de atención con orientación especifica a la atención de una población joven y con alto riesgo adicción.

- Capacitar y formar al equipo de enfermería materno Infantil para que identifique los factores de resiliencia de una adolescentes en riego de consumo, a los efectos de realizar un plan de cuidado personalizado y su oportuna derivación.

\section{AGRADECIMIENTOS}

Agradecemos a todos los docentes de la Escuela de Enfermería de Ribeirão Preto y a los treinta y tres participantes de nueve países latinoamericanos que participaron en el proceso de implementación de los programas, y también al Gobierno del Japón y al Programa de Becas de la OEA por su apoyo financiero y becas que posibilitaron la implementación del "I Programa Regional de Capacitación en Investigación para Enfermeros de América Latina".

6. Nadidona MG, Marks RKmm. Enfermería maternal. 3a ed. New York (USA): McGraw-Hill Internacional; 1996.

7. Borcardi MIB. Gravidez en la adolescencia el parto en cuanto espacio de miedo. [Tesis]. São Paulo (SP): Escola de Enfermagem/USP; 1997.

8. Munist M, Santos H, Kotliarenco. Manual de identificación y promoción de la Resiliencia en niños y adolescentes. Ginebra (SWZ): OPS/OMS Fundación W K Kellogg (ASDI); 1998. 